\title{
A team spectral inspection platform based on ASERA
}

\author{
Hailong Yuan, Yanxia Zhang, Yue Wu, Yajuan Lei, \\ Yiqiao Dong, Zongrui Bai, Guangwei Li, Haotong Zhang \\ and Yongheng Zhao
}

Key Laboratory of Optical Astronomy, National Astronomical Observatories, Chinese

Academy of Sciences, Beijing, China

email: yuanhl@bao.ac.cn

\begin{abstract}
Currently large sky area spectral surveys like SDSS, 2dF, and LAMOST, using the new generation of telescopes and observatories, have provided massive spectral data sets for astronomical research. Most of the data can be automatically handled with pipelines, but visually inspection by human eyes is still necessary in several situations, like low SNR spectra, QSO recognition and peculiar spectra mining. Using ASERA, A Spectrum Eye Recognition Assistant, we can set up a team spectral inspection platform. On a preselected spectral data set, members of a team can individually view spectra one by one, find the best match template and estimate the redshift. Results from different members will be gathered and merged to raise the team work efficiency. ASERA mainly targets the spectra of SDSS and LAMOST fits data formats. Other formats can be supported with some conversion. Spectral templates from SDSS and LAMOST pipelines are embedded and users can easily add their own templates. Convenient cross identification interfaces with SDSS, SIMBAD, VIZIER, NED and DSS are also provided. An application example targeting finding strong emission line spectra from LAMOST DR2 is presented.
\end{abstract}

Keywords. techniques: spectroscopic, surveys

\section{Introduction}

Nowadays massive celestial spectral data have been obtained from large sky area spectral surveys like SDSS (York et al. 2000), 2dF (Colless et al. 2001), and LAMOST (Wang et al. 1996). The latest data release 13 of the ongoing SDSS, which is the first data release of its fourth phase, has a total unique area coverage of 14,555 square degrees and 4,355,200 total spectra (See http://www.sdss.org/dr13/). About half of them are galaxies. The final data release of 2dF Galaxy Redshift Survey includes 269,937 non-unique spectra (See http://www.2dfgrs.net/). The majority are galaxies. The third data release of LAMOST has 5,755,126 spectra and 2,667 plates totally and more spectra will be obtained and released (See http://www.lamost.org/). Such abundant data will greatly promote astronomical research. Yet new algorithms and softwares with the capabilities for handling big data are in great need. Although many automatic data reduction and parameter estimation pipelines are developed, scientists may still need to inspect and analyse spectra one by one under the situation of getting good parameter estimation, flaw data processing, special characteristics hunting and so on. To meet those needs, we here provide a team spectral inspection platform based on ASERA (Yuan et al. 2013). In the following sections, we will introduce the basic spectral visualization, template matching and redshift estimation, parameter estimation, and team spectral inspection, 


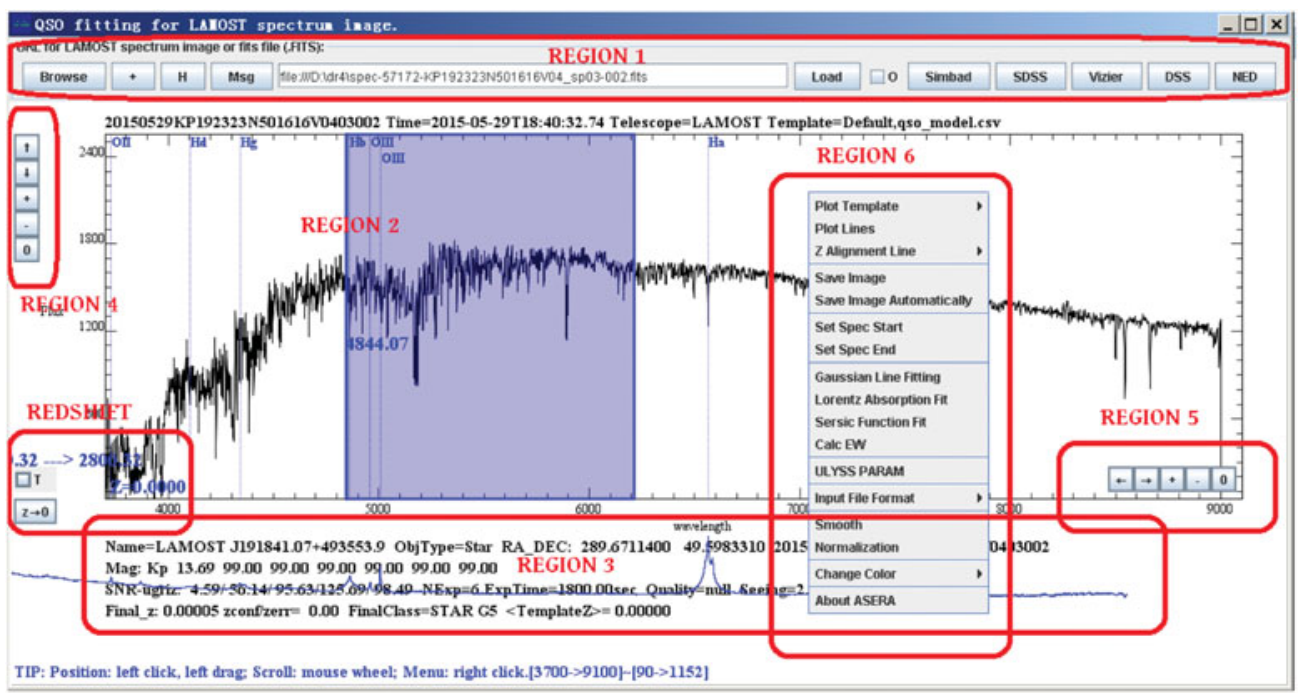

Figure 1. Main interface of ASERA.

separately. In the application of ASERA, several particular spectra of LAMOST are found.

\section{Spectral visualization}

Although there have been already dozens of astronomical spectral visualization softwares (e.g. VOSpec, SPLAT and SpecView), ASERA focuses on the visualization of the massive spectra from LAMOST and SDSS with basic necessary abilities, such as convenient axis specification, target information presentation, smoothing and user-defined colour. The main interface (Fig. 1) can be divided into several regions. Region 1 shows the spectral file URL and some import buttons. One can choose a bunch of spectral files at one time, from either local disk or the internet, and plot them one by one. Spectra from Simple Spectral Access Protocol (SSAP) server can be recognized but the embedded fits may vary among different survey projects. We currently support the spectral fits from SDSS and LAMOST. Tabular textual files containing two columns, the wavelength column and the flux column, are also supported so users need make transformation facing other data formats. In addition, general pixel images (e.g. JPG, PNG and GIF) can be loaded and compared with templates. On the right side of this region, several buttons are provided with the abilities of cross identification with SDSS, SIMBAD, DSS, Vizier and NED. Region 2 shows both the spectrum and the selected template. In view mode, one can drag a small region to zoom in and double click to reset the view port. In template match mode, one can intuitively change the redshift and flux scale of the template conveniently to find the best match between the target spectrum and the template. Region 3 displays the observation information of the target spectrum to help users understand the spectrum. Region 4 and 5 provide more specific flux and wavelength range adjustment abilities. Region 6 indicates the popup menu when users right click on the main view port. Menu items are grouped according to their functions. For example, shown in Fig. 1 is a G type spectrum from LAMOST, the wavelength range is from 3700 to 9100 angstroms, the $\mathrm{MgH}$ band and $\mathrm{Ca}$ triplet feature are apparent and the hydrogen lines are weak. 


\section{Template matching and redshift estimation}

Since the redshifts of QSOs cover a large range, the optical spectra of different redshifts vary from each other. Thus the automatic template matching classification and redshift measurement pipelines can't obtain reliable results especially when the spectral quality is poor. However ASERA exactly provides a platform to help users compare the target spectra with templates under different redshifts and then determine the redshift of the target spectra, taking full advantages of the experiences from astronomical experts. Besides the QSO templates, ASERA also provides 33 spectral templates from SDSS pipeline and 182 templates from LAMOST, including various types of stars, galaxies, white dwarfs, binaries, cataclysmic variables and so on (Yuan et al. (2014)). In addition, users can add their own specialized spectra as templates and the detailed examples are shown in http://lamostss.bao.ac.cn/ yhl/asera. Considering the redshifts for stars are small, the non-QSO templates should be only used to give rough redshift estimation by this way. Meanwhile ASERA owns additional functions like line fitting, line equivalent width measurement. By integrating UlySS, ASERA may obtain reliable stellar parameter estimation. Moreover using batch process mode, users can inspect a large sample efficiently.

\section{Team spectral inspection}

Considering the fact that more than ten million optical spectral data are released from SDSS and LAMOST and more data will be obtained in the future, it is necessary to create a whole team of the same interest and perform the eyeball inspection on very large number of spectra. ASERA is very suitable to meet this need. The whole platform serves as a Server/Client mode. The spectral data and inspection results are stored and published via MySQL database and web server software. Any interested user can connect to the server using ASERA and fetch a subset of spectra that haven't been inspected. The team administrator should monitor and manipulate the whole work process under the MySQL environment. Generally four things are needed to start the work: 1) setup a web server that provides the team members access to all the spectral files; 2) setup a database table containing all the necessary columns about spectral files to help select wanted spectral files, like RA, DEC, SNR and inspection records. The additional special features are provided as required; 3) setup a basic ASERA feedback table to store the template, redshift and user comments for each spectrum; 4) setup a ULYSS feedback table to store $T_{\text {eff }}, \log g,[\mathrm{Fe} / \mathrm{H}]$ and $R V$ for each spectrum.

For the client side, users need to do three things: 1) enable ASERA feedback by providing a configuration file; 2) enable ULYSS feedback by providing a configuration file; 3) input properly the "MySQL DB", "URL pattern" and "Query string" to access spectral files. Then the team starts to work with ASERA by the batch process mode discussed above. By optimizing the SQL query, users can make things going on in the way as wished. For example, celestial object classification may be determined from several line indexes, then based on the classification information, clients firstly inspect those unchecked data with reliable classification.

\section{Example spectra from LAMOST}

Here some spectra observed by LAMOST are shown in Fig. 2, including 2 cataclysmic variables, 1 galaxies, 1 QSOs, 1 M-type emission line star and 1 T Tauri star. The SNR of these spectra are poor and classification from the current pipeline may be debatable. 


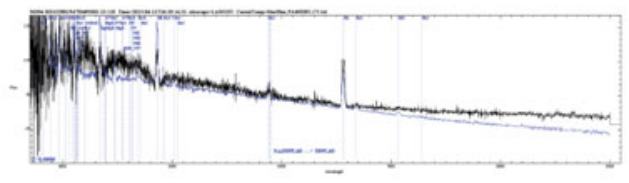

(a) 20130411HD132901N475049M0113119

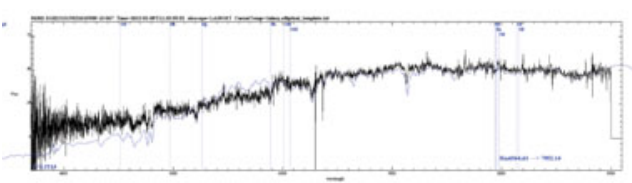

(c) 20130109EG023131N032619F0915067

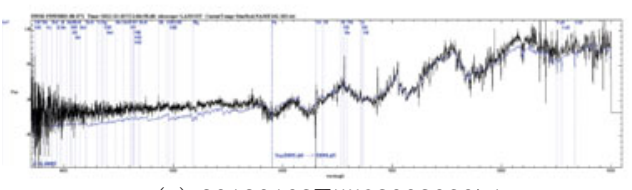

(e) 20120103F559300308071

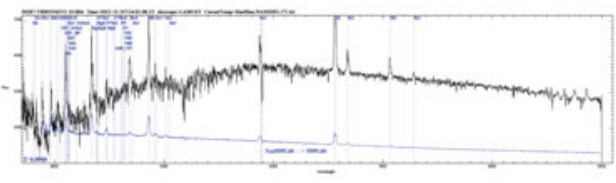

(b) 20121225VB053N42V215004

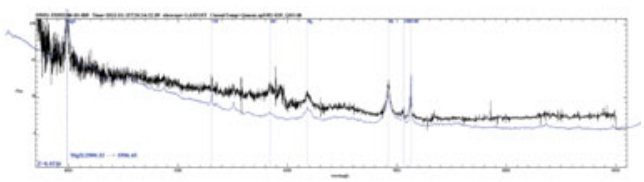

(d) 20120125F559520603009

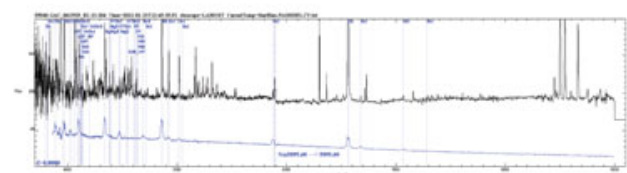

(f) 20120113GAC_061N29_B113204

Figure 2. Example spectra from LAMOST

However their distinct features can be confirmed easily via human inspection and then better classification will correctly given. If these spectra are used as templates, a peculiar spectra hunting project may be performed with a team of interest on massive spectral data.

\section{Acknowledgements}

This paper is funded by the National Natural Science Foundation of China under grant No.11403046. The LAMOST FELLOWSHIP is supported by Special Funding for Advanced Users, budgeted and administrated by Center for Astronomical Mega-Science, Chinese Academic of Sciences (CAMS).

\section{References}

York, D. G., Adelman, J., Anderson, J. E., Jr., et al. 2000, AJ, 120, 1579

Colless, M., Dalton, G., Maddox, S., et al. 2001, MNRAS, 328, 1039

Wang, S.-G., Su, D.-Q., Chu, Y.-Q., Cui, X., \& Wang, Y.-N. 1996, Applied Optics, 35, 5155

Yuan, H., Zhang, H., Zhang, Y., et al. 2013, Astronomy and Computing, 3, 65

Yuan, H., Zhang, H., Zhang, Y., et al. 2014, ADASS XXIII proceeding edited by N. Manset and P. Forshay, 485, 455 